\title{
Análisis del consumo de la quinua y sus derivados en el cantón Riobamba
}

\section{Analysis of the consumption of quinoa and its derivatives in the Riobamba canton}

1 Eduardo Xavier Centeno Parra https://orcid.org/0000-0003-4311-7228

Escuela Superior Politécnica de Chimborazo (ESPOCH); Dirección de Evaluación y Aseguramiento de la Calidad; Chimborazo xavier.centeno@espoch.edu.ec

2 Jose Gabriel Pilaguano Mendoza https://orcid.org/0000-0002-7153-6879 Escuela Superior Politécnica de Chimborazo (ESPOCH). Carrera de Finanzas; Chimborazo gpilaguano@espoch.edu.ec

3 Jorge Luis Chafla Granda https://orcid.org/0000-0003-2299-7430 Escuela Superior Politécnica de Chimborazo (ESPOCH). Carrera Finanzas; Chimborazo; jorge.chafla@espoch.edu.ec

4 Jessica Ernestina Duran Delgado https://orcid.org/0000-0003-3740-1601 Universidad Estatal de Bolívar (UEB), Carrera Ingeniería Comercial; Bolívar; jessica.duran@ueb.edu.ec

Artículo de Investigación Científica y Tecnológica Enviado: 12/12/2021

Revisado: $27 / 12 / 2021$

Aceptado: 14/01/2022

Publicado:07/02/2022

DOI: https://doi.org/10.33262/concienciadigital.v5i1.2062

Cítese: $\quad$ Centeno Parra, E. X., Pilaguano Mendoza, J. G., Chafla Granda, J. L., \& Duran Delgado, J. E. (2022). Análisis del consumo de la quinua y sus derivados en el cantón Riobamba. $5(1)$ 118-129. https://doi.org/10.33262/concienciadigital.v5i1.2062

CONCIENCIA DIGITAL, es una Revista Multidisciplinar, Trimestral, que se publicará en soporte electrónico tiene como misión contribuir a la formación de profesionales competentes con visión humanística y crítica que sean capaces de exponer sus resultados investigativos y científicos en la misma medida que se promueva mediante su intervención cambios positivos en la sociedad. https://concienciadigital.org

La revista es editada por la Editorial Ciencia Digital (Editorial de prestigio registrada en la Cámara Ecuatoriana de Libro con No de Afiliación 663) www.celibro.org.ec

Esta revista está protegida bajo una licencia Creative Commons AttributionNonCommercialNoDerivatives

4.0 International. Copia de la licencia: http://creativecommons.org/licenses/by-nc-nd/4.0/ 


\section{Palabras claves:}

quinua,

producción, comercialización, exportación, estadística,

Riobamba.

\section{Keywords:}

quinoa, production, marketing, export, statistics, Riobamba.
Resumen

En Ecuador la producción y exportación de la quinua se ha incrementado en los últimos años, por otro lado, al analizar el consumo de este grano integral y sus derivados se ha notado que el desconocimiento de sus propiedades ha permitido que las personas no consuman dicho producto. El objetivo a seguir es el análisis de la conceptualización del origen, propiedad y consumo de la quinua. Metodología: Aplicamos una metodología del Diseño no experimental, diseño transeccional, transeccionales descriptivos, además se articulan con las técnicas cualitativa y cuantitativa construyendo de esta manera herramientas para realizar un análisis estadístico, en los resultados por medio de la muestra se llegará a la tabulación y disertación de los datos obtenidos que nos permitirán diseñar estrategias, dando de esta manera la conclusión del estudio propuesto que con diferentes alternativas se puede mejorar y fomentar la comercialización de la quinua y sus derivados.

\section{Abstract}

In Ecuador, the production and export of quinoa has increased in recent years, on the other hand, when analyzing the consumption of this whole grain and its derivatives, it has been noted that ignorance of its properties has allowed people not to consume said product. The objective to follow is the analysis of the conceptualization of the origin, property and consumption of quinoa. Methodology: We apply a methodology of nonexperimental design, transactional design, descriptive transactional, in addition they are articulated with qualitative and quantitative techniques, thus building tools to carry out a statistical analysis, in the results through the sample the tabulation will be reached and Dissertation of the data obtained that will allow us to design strategies, thus giving the conclusion of the proposed study that with different alternatives it is possible to improve and promote the commercialization of quinoa and its derivatives.

\section{Introducción}

El análisis realizado se toma en cuenta las bases teóricas de la producción de la quinua para poder determinar necesidades, que puedan plantarse en este estudio. 
En el Ecuador según Tapia (1979), la quinua ha persistido entre los campesinos del área de Carchi, Imbabura, Pichincha, Cotopaxi, Chimborazo y Loja (Cardozo et al., 1976). Considerando esta productividad que se da en el país, se ratifica que es uno de los mayores productores a nivel de Latinoamérica, como consecuencia el consumo de los productos derivados de la quina esta en proyección hacia arriba.

Morales (1975), comparó unos 18 eco tipos provenientes de las zonas de Imbabura, Cayambe, Cotopaxi y Chimborazo con material de Bolivia y destacaron los eco tipos ecuatorianos "Chaucha", "Punín", "Grande" y "Staquinua" con muy buenos rendimientos.

Es importante observar que los eco tipos locales varían en su periodo de crecimiento que va de 170 a 227 días y que la variedad Sajama traída de Bolivia, completa su maduración en 123 días, por efecto del cambio de fotoperiodo. Los rendimientos de Sajama fueron muy bajos, sin embargo, se debe anotar que la susceptibilidad de esta variedad al "mildiú" y a la bacteriosis es muy alta en medios húmedos como la sierra del Ecuador.

Las quinuas de Latacunga, Ambato, Carchi, Riobamba y Cuenca son de grano chico, en general de porte elevado y de grano bastante amargo. Se calcula que la superficie total cultivada es de unas 1200 ha en todo el país (Freire et al., 1975).

Según Peralta y Mazón (2014), el Ecuador produce una cantidad muy inferior de quinua en comparación a Bolivia y Perú; no obstante, el país tiene un rendimiento promedio de 30 a 50\% más alto que estos países. La competitividad futura del Ecuador podrá depender no solo de la capacidad de aumentar el área bajo producción, sino más bien de aumentar la productividad, calidad y reconocimiento de esta producción (Jacobsen \& Sherwood, 2002).

Junovich (2003), en base de los resultados del III Censo Agropecuario realizado en el año 2000 informa que para la quinua se registraron 2659 Unidades de Producción Agropecuaria (UPAs), con cerca de 900 ha sembradas, habiendo sido cosechadas 636 ha y con una producción total de 226 t, de las cuales se vendieron 180 t. El rendimiento promedio para la Sierra es de 0,4 t/ha. El promedio de superficie sembrada con quinua es de 0,3 ha/UPA. Las provincias con mayor número de UPAs con quinua son Chimborazo, Cotopaxi e Imbabura; pero la principal provincia productora es Chimborazo, en donde se obtuvo (en el período censal) cerca del $80 \%$ de la producción total.

En los últimos años el área sembrada de quinua en Ecuador ha fluctuado entre menos de 500 ha hasta alrededor de 1200 ha/año, que significan volúmenes no mayores a las 500 $600 \mathrm{t}$ (figura 1). 


\section{Figura 1}

Producción de quinua por país en el periodo 1990-2012

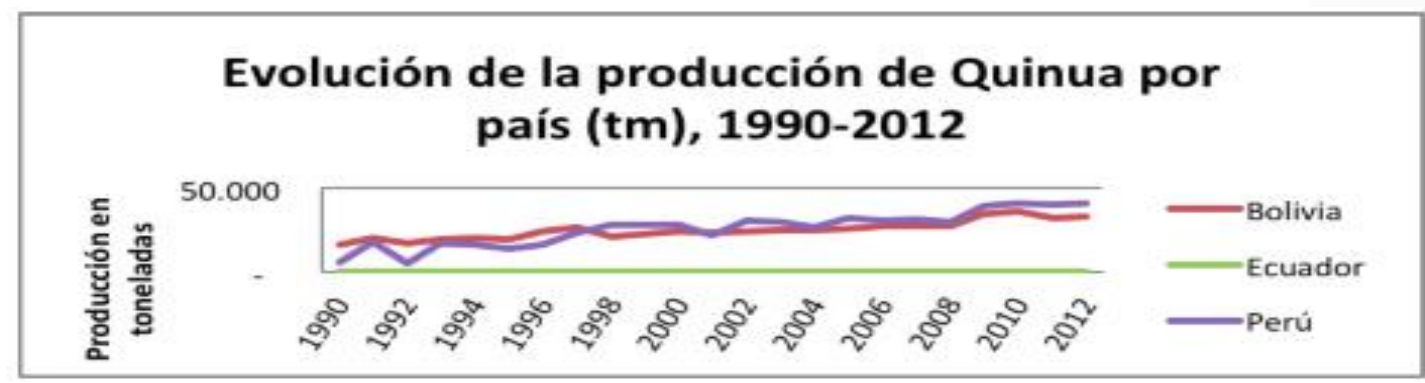

Fuente: Tomado de Ministerio de Agricultura, Ganadería y Pesca (MAGAP, 2013)

\section{Figura 2}

Importaciones de quinua al Ecuador

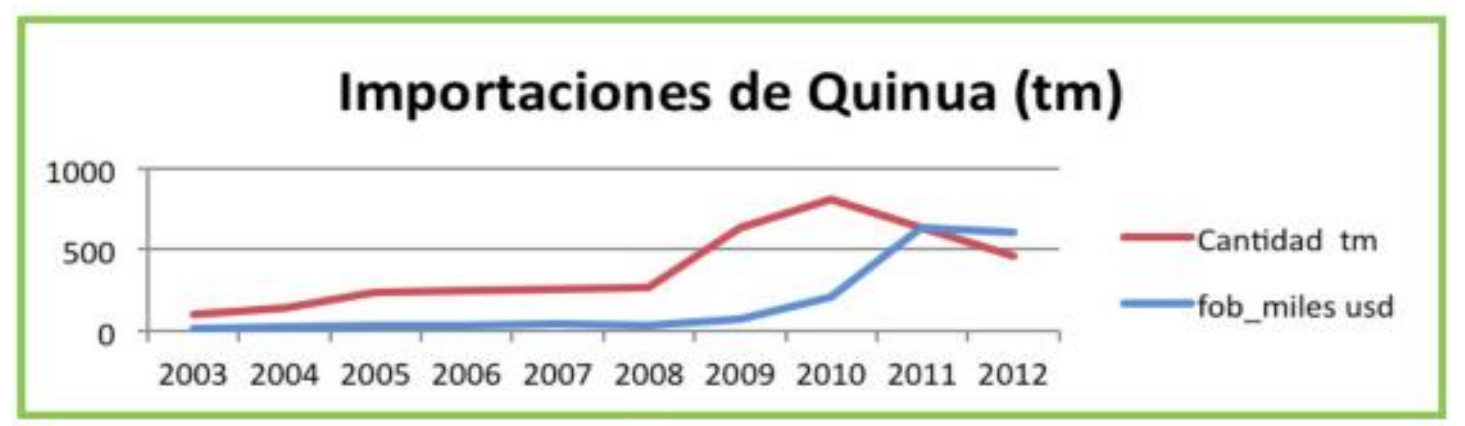

Nota: Las importaciones de quinua al Ecuador, desde el año 2009 han sido superiores a las 500 t/año. Fuente: Tomado de MAGAP (2013)

Según el proyecto de fomento de la quinua del MAGAP, las exportaciones de quinua desde el Ecuador, no han superado las 500 t/año (Figura 3). Según el Banco Central, en el Ecuador las exportaciones de quinua presentan fluctuaciones desde 1987; el análisis de los últimos 8 años permite determinar un importante incremento comparando las 41 toneladas exportadas en el 2000 con las 422 toneladas en el 2008. Entre el 2004-2005 estas se han mantenido relativamente constantes, a pesar de que en el año 2006 se registra un descenso del $18 \%$ en relación al 2005. Igualmente, el precio FOB, ha experimentado un incremento, registrándose el mayor valor por tonelada (USD 1870,8) en el año 2008. 


\section{Conciencia}

\section{Figura 3}

Exportaciones de quinua de Ecuador

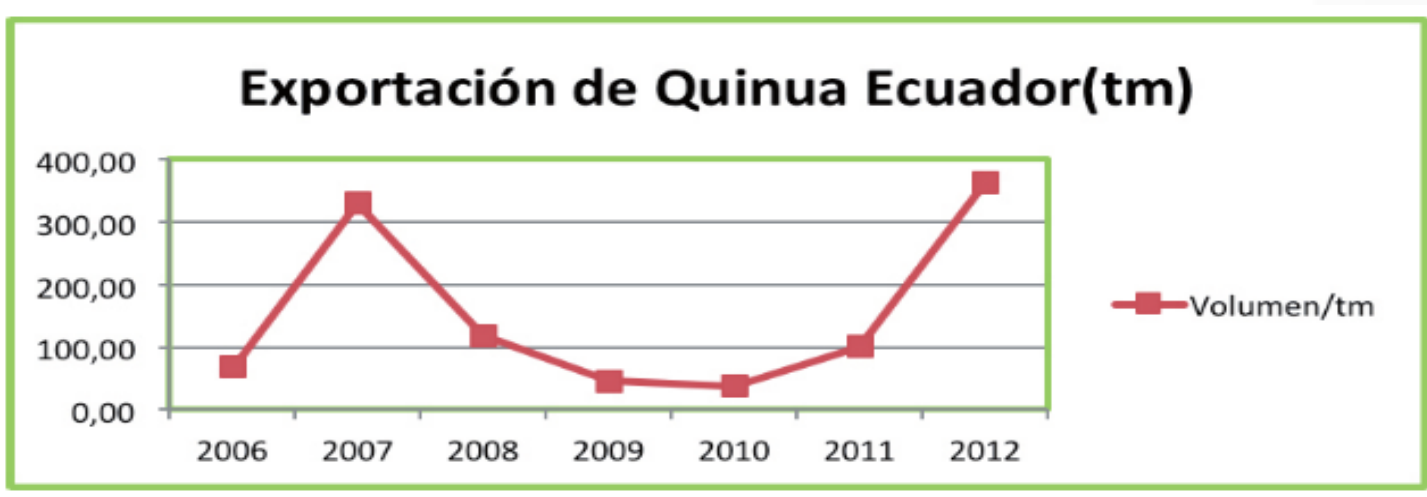

Fuente: Tomado de MAGAP (2013)

\section{Figura 4}

Demanda anual de la quinua ecuatoriana, período 2000 - 2008.

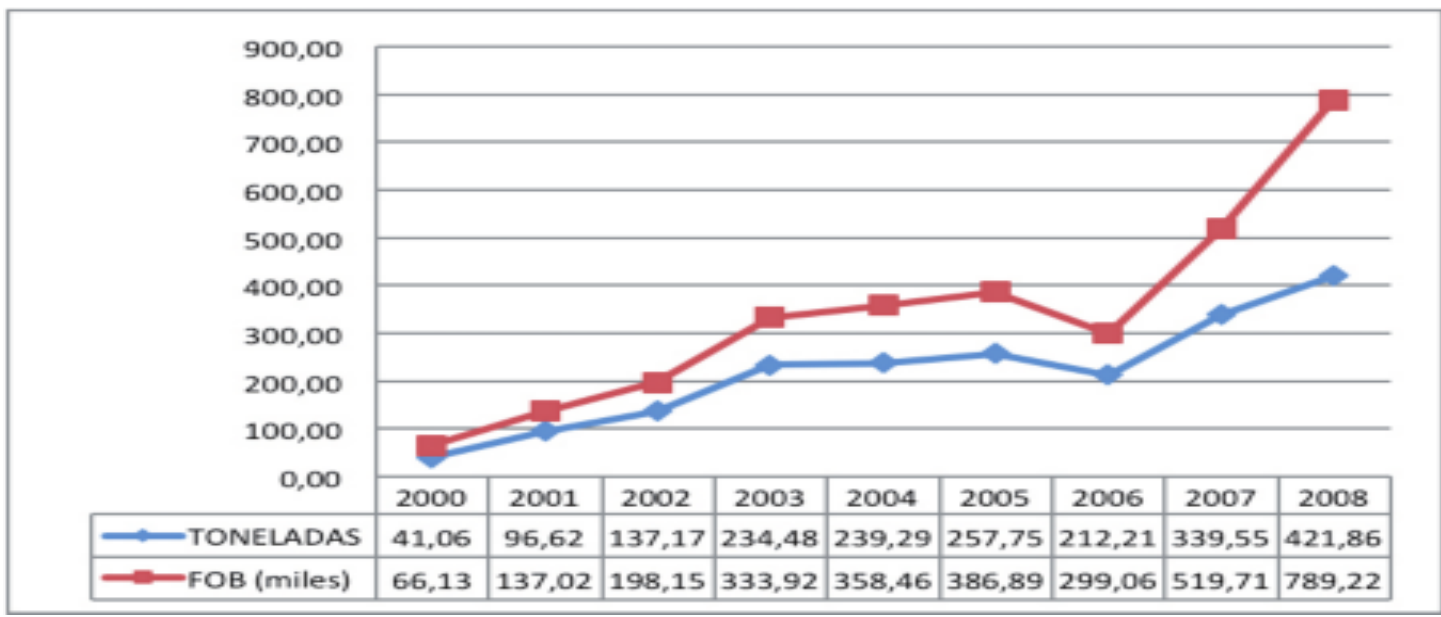

Fuente: Tomado de Banco Central del Ecuador (BCE, 2009)

Durante el período 2000-2008 los principales destinos de la quinua ecuatoriana han sido Estados Unidos con el 53\%, el Reino Unido con el 29\%, Francia el 6\%, Alemania y España el $4 \%$ y otros países a los que corresponde el $4 \%$ de las exportaciones totales (figura 5). 


\section{Figura 5}

Principales países de destino de la quinua ecuatoriana, período 2000 - 2008

\section{INIAP - Estación Experimental Santa Catalina}
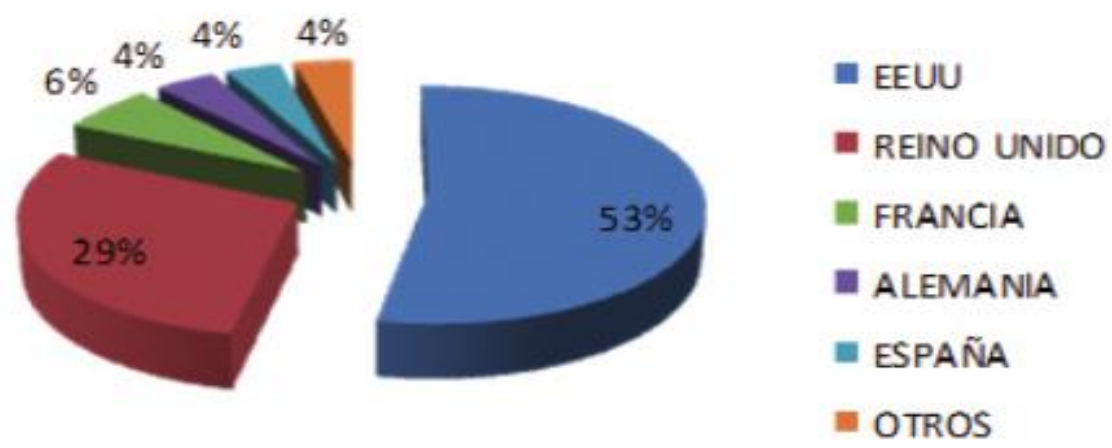

Nota: El consumo de quinua se encuentra limitado, por el desconocimiento de las buenas cualidades nutritivas, otro obstáculo constituye el lavado que se necesita para que el grano pueda ser consumido sin embargo el consumo está al alza por cuestiones de alimentación y proteína. Fuente: Tomado de Banco Central del Ecuador (BCE, 2009)

\section{Metodología}

El diseño no experimental es aquel que se realiza sin manipular deliberadamente variables. Se basa fundamentalmente en la observación de fenómenos tal y como se dan en su contexto natural para después analizarlos.

Su propósito es describir variables y analizar su influencia e interrelación en un momento dado.

Pueden abarcar varios grupos o subgrupos de personas, objetos o indicadores y se pueden dividir en 3 tipos:

- Transeccionales Exploratorios

- Transeccionales Descriptivos

- Transeccionales Correlaciónales-casuales

\section{Transeccionales Descriptivos}

Recolectan datos sobre cada una de las categorías, conceptos, variables, contextos y reportan los datos que obtienen (Dzul, 2013). 
Para la realización de la presente investigación se utilizó los dos tipos de enfoque cualitativo y cuantitativo.

El enfoque cualitativo según Rodríguez (2011):

Utiliza la recolección de datos sin medición numérica para descubrir o afinar preguntas de investigación en el proceso de interpretación. EI enfoque se basa en métodos de recolección de datos no estandarizados. No se efectúa una medición numérica, por lo cual el análisis no es estadístico.

EI enfoque cualitativo puede definirse como un conjunto de prácticas interpretativas que hacen al mundo visible, lo transforman y convierten en una serie de representaciones en forma de observaciones, anotaciones, grabaciones y documentos. (pp. 01-02)

Para la siguiente investigación se realizó mediante el enfoque cualitativo donde se procedió a construir herramientas como encuestas que aplicadas a la muestra en el cantón Riobamba, donde se consideró las opiniones de las personas sobre la comercialización y consumo de la quinua.

Según Rodríguez (2011), el enfoque cuantitativo:

Usa la recolección de datos para probar hipótesis, con base en la medición numérica y el análisis estadístico, para establecer patrones de comportamiento y probar teorías. Este enfoque utiliza la lógica o razonamiento deductivo, que comienza con la teoría y de esta se derivan expresiones lógicas denominadas hipótesis que el investigador busca someter a prueba. Se signe rigurosamente el proceso $\mathrm{y}$, de acuerdo con ciertas reglas lógicas, los datos generados poseen los estándares de validez y confiabilidad, las conclusiones derivadas contribuirán a la generación de conocimiento. (pp. 04-05)

El enfoque cuantitativo nos permite presentar el análisis de los datos en representación de tablas, gráficos, análisis de forma numérica y porcentual para una mejor comprensión de los datos. Según Ventura-León (2017), la población es un conjunto de elementos que contienen ciertas características que se pretenden estudiar. Por esa razón, entre la población y la muestra existe un carácter inductivo (de lo particular a lo general), esperando que la parte observada (en este caso la muestra) sea representativa de la realidad.

Al proceder con la investigación se consideró una muestra aplicada al cantón Riobamba según el Instituto Nacional de Estadística y Censos (INEC, 2010), la población urbana es de 3900 consumidores económicamente activos donde se utilizó un nivel de confianza del $95 \%$ y un $5 \%$ de margen de error. 


\section{Resultados}

Se considera como muestra a 350 personas, las mismas que fueron encuestadas por medio de Google Forms y de las cuales 130 pertenecen al género masculino y 220 al género femenino es decir el $37.14 \%$ y $62.86 \%$ respectivamente, la finalidad de esta actividad es recabar información real y acertada con respecto al conocimiento, consumo, usos, beneficios y precio de la quinua, esto es sumamente importante ya que a través de estos datos se podrán plantear y establecer estrategias para incentivar e incrementar el consumo de quinua.

A continuación, se reflejan las variables investigadas:

Con respecto a la variable consumo de la Quinua de las 350 personas encuestadas por medio de Google formularios, se determinó que $63.43 \%$ Si consumen la quinua, el $29.43 \%$ no consumen la quinua y el $1.14 \%$ no saben consumir la quinua.

Conoce las variedades de la Quinua De las 350 personas encuestadas por medio de Google formularios, se determinó que $28.29 \%$ Si conocen las variedades de la quinua, el $67.14 \%$ no conocen las variedades la quinua y el $4.57 \%$ se preguntan cuáles son las variedades de la quinua.

Recomendaría usted consumir la quinua a los demás De las 350 personas encuestadas por medio de Google formularios, se determinó que $93.43 \%$ Si recomiendan el consumo de la quinua, el $6.57 \%$ no recomiendan el consumo de la quinua.

Con respecto a la variable que usted los diferentes platos de preparación de la quinua de las 350 personas encuestadas por medio de Google formularios, se determinó que $48.86 \%$ Si tienen conocimiento de las distintas preparaciones de la quinua, el $46.00 \%$ no tienen conocimiento de las diferentes preparaciones de la quinua y el $5.14 \%$ se preguntan cuáles son los diferentes tipos de preparación de la quinua.

Con respecto a la variable le parece a usted agradable el sabor de la quinua de las 350 personas encuestadas por medio de Google formularios, se determinó que $79.71 \%$ Si les parece agradable el sabor la quinua, el $20.29 \%$ no les parece agradable el sabor de la quinua.

Con respecto a la variable conoce usted como se cultiva la quinua de las 350 personas encuestadas por medio de Google formularios, se determinó que 28,86\% Si tienen el conocimiento de cómo se cultiva la quinua, el $71.14 \%$ no tienen el conocimiento de cómo se cultiva la quinua.

Con respecto a la variable conoce usted las características de la quinua de las 350 personas encuestadas por medio de Google formularios, se determinó que $28,86 \% \mathrm{Si}$ tienen el conocimiento de las características la quinua, el 58\% no tienen el conocimiento 
de las características de la quinua y el 4,29\% se preguntan cuáles son las características de la quinua.

Según la variable conoce las propiedades y beneficios que brinda la quinua De las 350 personas encuestadas por medio de Google formularios, se determinó que $48.57 \% \mathrm{Si}$ tienen el conocimiento de las propiedades y beneficios la quinua, el $46.57 \%$ no tienen el conocimiento de las propiedades y características de la quinua y el $4.86 \%$ se preguntan cuáles son las propiedades y características de la quinua.

Según la variable donde suele comprar la quinua de las 350 personas encuestadas por medio de Google formularios, se determinó que el 37.43\% compran la quinua en el supermercado, el $0.86 \%$ compran la quinua mediante el internet, el 53.14 compran la quinua en mercados y el $8.57 \%$ compran la quinua directamente del productor.

Según la variable tiene alguna preferencia especial en cuanto a la marca de la quinua que consume De las 350 personas encuestadas por medio de Google formularios, se determinó que el $16.57 \%$ prefieren consumir la quinua industrializada (Empaquetada), el $22 \%$ prefieren consumir la quinua a granel, y el $61.43 \%$ no prefieren consumir la quinua en ninguna presentación.

Según la variable conoce usted el valor de la quinua de las 350 personas encuestadas por medio de Google formularios, se determinó que el $47.43 \%$ si conocen el valor de la quinua, el $48.29 \%$ no conocen el valor de la quinua, y el $4.29 \%$ preguntan cuál es el valor de la quinua.

Según la variable cuanto estaría dispuesto a pagar por una libra de quinua de las 350 personas encuestadas por medio de Google formularios, se determinó que el 59.43\% está dispuesto a pagar entre 0,50 a 1,00 por una libra de quinua, el $32.57 \%$ está dispuesto a pagar entre 1,00 a 1,50 por una libra de quinua, el $6.29 \%$ está dispuesto a pagar entre 1,51 a 2,00 por una libra de quinua y el $1.71 \%$ está dispuesto a pagar entre 2,01 a 2,50 por una libra de quinua.

Al final se ha obtenido los resultados establecidos de la investigación ya que por medio del análisis de las variables a grupo de personas nos ha permitido obtener información importante para determinar estrategias para incrementar el consumo de la quinua y sus derivados

\section{Conclusiones}

- En Chimborazo existe una productividad de quinua bastante óptima y a la vez acogida por las personas si se desea comercializar o ampliar en el mercado tanto nacional como internacional tendría buenas posibilidades de éxito, la mayoría de la población conoce de la quinua, pero de igual manera existe un grupo de 
personas que no, lo cual es favorable ya que es un público objetivo para pretender consumir sus derivados, al mismo tiempo se puede recomendar el consumo de la misma.

- Que las asociaciones capaciten o socialicen el proceso orgánico de cultivo y los beneficios de la quinua a través de una campaña de comunicación a la sociedad con el fin de concientizar la importancia la misma.

- Dentro del estudio de mercado se puede ver que en la localidad los diferentes miembros de las organizaciones tienen en un $92,86 \%$ conocimiento de la quinua, valor que se complementa con un número considerable del $69,43 \%$ de consumo de la quinua. Se puede analizar además que existe en un $93,43 \%$ de las personas que consumen lo recomiendan para consumo.

- En cuanto a la diversificación del consumo en un 48,86\% tienen un conocimiento por fuera del tradicional sobre los usos y preparaciones alimenticias que se pueden derivar de la quinua como producto principal para la cocina. Un tema minoritario pero que puede tener gran impacto es la apreciación del sabor a pesar de que en un $20,29 \%$ se tiene una percepción negativa del sabor debe ser un síntoma de análisis en futuros estudios para poder contemplar las razones por las cuales se tiene este malestar en el consumo de la quinua.

- Existe en el mercado de consumo de la quinua un gran desconocimiento hacia los métodos productivos del producto, con un $28,86 \%$ es la porción de las personas que se encuesto conocen puntualmente el método productivo. Por el contrario, existe un empate técnico al momento de hablar sobre las propiedades y beneficios de la quinua dado que en un $48,57 \%$ se tiene conocimiento sobre la parte positiva y propiedades alimenticias de la quinua y un porcentaje similar del $46,57 \%$ no tiene conocimiento sobre este hecho.

- Finalmente, las preferencias para el consumo de este producto alimenticio son en un $53,14 \%$ los mercados y en un $37,43 \%$ los supermercados. Se debe mencionar que en el proceso de agregación de valor no existe ni marcas ni formas de conservación que se encuentren en la mente del consumidor y el valor para compra de una libra de quinua en un 59,43\% se concentra entre los USD 0,50 a 1,00.

\section{Referencias Bibliográficas}

Banco Central del Ecuador [BCE]. (2009). Demanda anual de la quinua ecuatoriana periodo 2000-2008. https://contenido.bce.fin.ec/home1/estadisticas/bolmensual/IEMensual.jsp

Cardozo, A., Vizcarra, I., Naranjo, M., Tapia, M., \& Rea , J. (1976). Bibliografía Internacional de Quinua y Cañahua. Bogotá: IICA. 
Dzul Escamilla, M. (2013). Aplicación básica de métodos científicos, método no experimental. México: Universidad Autónoma de Estado de Hidalgo. https://www.uaeh.edu.mx/docencia/VI_Presentaciones/licenciatura_en_mercado tecnia/fundamentos_de_metodologia_investigacion/PRES38.pdf

Freire, M., Rea, J., \& Rijks, J. Q. (1975). Estudio agro climatológico de la zona andina. Roma.

Instituto Nacional de Estadística y Censos [INEC]. (2010). Población del Cantón Riobamba Censo 2001. Obtenido de https://www.ecuadorencifras.gob.ec/documentos/webinec/Bibliotecas/Fasciculos_Censales/Fasc_Cantonales/Chimborazo/Fasciculo_ Riobamba.pdf

Jacobsen, S., \& Sherwood, S. (2002). Cultivo de Granos Andinos en Ecuador: Informe sobre los rubros quinua, chocho y amaranto. Quito: Ediciones Abaya-Yala.

Junovich, A. (2003). La Quinua en el Ecuador a través de los datos del III Censo Agropecuario. Quito: Proyecto SICA-Banco Mundial.

Ministerio de Agricultura, Ganadería y Pesca [MAGAP]. (2013). Proyecto de fomento de la producción de quinua en Ecuador. https://repositorio.iniap.gob.ec/bitstream/41000/2433/1/iniapscCD13.pdf

Morales, M. (1975). Comportamiento, agronomía y análisis bromatológico de 20 eco tipos de quinua (Chenopodium quinoa Willd.) en Cayambe, Pichincha. Quito.

Peralta, E., \& Mazón, N. (2014). La quinua en Ecuador. Santiago de Chile: FAO. http://repositorio.iniap.gob.ec/handle/41000/2433

Rodríguez, J. (2011). Métodos de investigación cualitativa. Revista de Investigación Silogismo, 08.

Tapia, M. (1979). La quinua y la kañiwa: Cultivos Andinos. Bogotá: Orton IICA.

Ventura-León, J. L. (octubre de 2017). ¿Población o muestra?: Una diferencia necesaria. Revista Cubana de Salud Pública, 43(04). http://scielo.sld.cu/scielo.php?script=sci_arttext\&pid=S086434662017000400014

\section{Ciencia Ligital}




\section{Conciencia}

El artículo que se publica es de exclusiva responsabilidad de los autores y no necesariamente reflejan el pensamiento de la Revista Conciencia Digital.

\section{LCiencia}

El artículo queda en propiedad de la revista y, por tanto, su publicación parcial y/o total en otro medio tiene que ser autorizado por el director de la Revista Conciencia Digital.
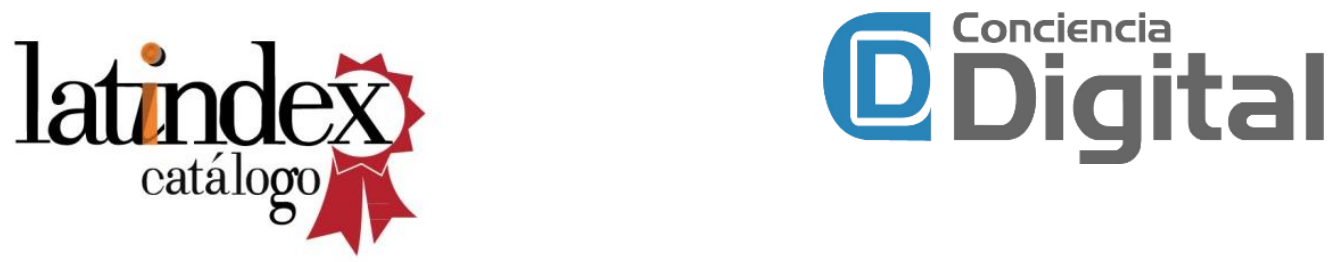

Indexaciones

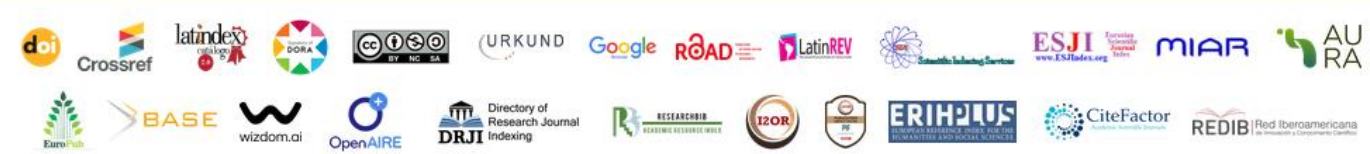

